\title{
Metaphor in English Advertisement - Analysis Based on the Conceptual Integration Theory
}

\author{
Yang Zhishang \\ Changsha Medical University, Changsha, Hunan,413000 China \\ 284204813@qq.com
}

Keywords: pun of advertisement; conceptual integration; cognitive interpretation

\begin{abstract}
Conceptual integration is a general cognitive activity of human beings. The essay discussed relevant mental mechanisms of readers or audiences when understanding puns and intentions of advertisements by use of the conceptual integration theory of cognitive linguisticians like Fauconnier and by integrating network through conceptual integration. The research indicated that the conceptual integration theory can describe and illustrate the understanding process of puns of advertisements in detail. It is a new perspective of study on advertisement puns to understand advertisement puns from the aspect of conceptual integration and it is a significant attempt.
\end{abstract}

\section{Introduction}

Advertisement language is a special language. To attract attention of consumers to products advertised and stimulate their buying inclination and promote their consuming behaviors in the end, a successful advertisement generally should be novel, vivid and appealing and can give thinking and imaginary space in language. Among rhetorical devices applied by advertisement creators to enhance language artistry of advertisements, pun is favored by most advertisers. Pun is a kind of work game which uses phonetic or semantic conditions to make a same linguistic form have dual meanings in a certain context. To discuss relevant metal mechanisms of readers or audiences when understanding advertisement puns from the cognitive perspective is to figure out what cognitive procedures are operated in human brains to understand real intentions of advertisement creators and integrate the cognitive process to represent it dynamically in the form of network diagram.

\section{Metaphor and conceptual integration}

The conceptual integration network is constituted by four psychological spaces: two input spaces with one belonging to generic space and the other belonging to blended space, and they have corresponding elements. Through cross-space mapping, the abstract structure and information of the two spaces will be projected in generic space, and through partial mapping and matching, structures and corresponding elements of the two input spaces will be selectively projected in the blend space. In this way, the four psychological spaces will be combined to be a systematic conceptual integration network through cross-space mapping and projection, which is as shown in figure 1 . Since concepts of generic space do not involve cognitive treatment of conceptual integration, the essay would not include an analysis on generic space. In the blend space, partial structures and corresponding elements which are extracted from the two input spaces will e integrated into an emergent structure. As a core part of conceptual integration, emergent structure has new structures which are differing from other spaces. However, the emergent structure is not directly constituted by structures and information extracted from the two input spaces; instead, its building-up process is very complicated and includes three basic methods: combination, completion and elaboration. Combination refers to that conceptual contents of two or above input spaces combine a space combination, during when there will be new relations between input spaces. Completion refers to that when projecting structures from input spaces and information structures from long-term memory can match with each other, activated modes in the blend space such as schematic knowledge, cognition and cultural pattern will be completed. And elaboration means that structures of blend space can be expanded infinitely 
through cognitive operation according to emergent logic of the emergent structure. The three processes can reflect dynamics and complexity of emergent structure. In practical analysis procedures, the essay will fully consider the two properties of emergent structure and realize conceptual integration from the view point of context.

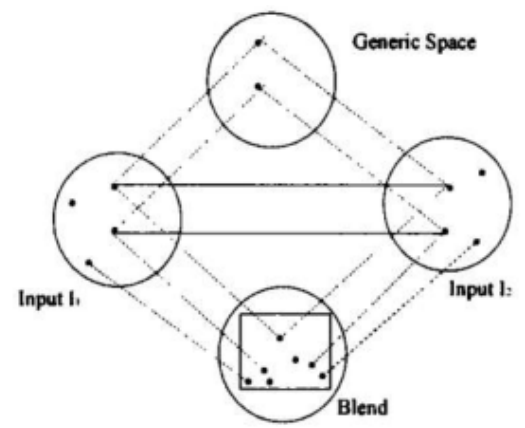

Figure 1 Working course of narrative language of news

\section{Case analysis of metaphor in print advertisements}

According to visual metaphor classification proposed by Forceville, after distinguishing visual metaphor categories of collected print perfume advertisements and analyzing various visual metaphors in print perfume advertisements through the conceptual integration theory, the essay discovered the characteristics of cognitive structures. After discrimination and analysis, the writer discovered that metaphor of print advertisements had another category except for pictorial simile, hybrid metaphor, contextual metaphor and integrated/ verbo-pictorial metaphor founded by Forceville $(1994,1996)$, and the writer named it pure contextual metaphor.

Advertisements with vivid simile. Among print perfume advertisements with vivid simile, target domain and source field can be clearly represented in advertisements, generally separately, which is as shown in figure 2. In advertisements, perfumes and females which are target domain and source field respectively are represented in print advertisements separately with tan unified background. The print advertisement compares perfume to beautiful women and its linguistic expression is "perfume is like female". However, the conceptual metaphor theory only explains results of consumers' cognizing the metaphor of the advertisement but not illustrates generative process of metaphor or cognitive characteristics of advertisements. Thus we can see that the two metal spaces, perfume and woman, have similarities in color and appearance. Through mapping and projection of identity, blend space will generate an emergent structure of characteristics of perfume, thus original similarities of the two input spaces will be retained and features of females will be involved in characters of perfume to transfer the meaning that perfume is as charming and moving as females. Moreover, the background of the two spaces is integrated into the blend space through projection to express the meaning of luxury and elegant, which is as shown in figure 3. The integrated conceptual network belongs to one-dimensional network.

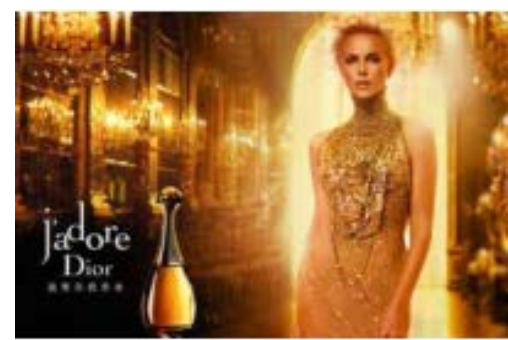

Figure 2 Advertisement with vivid simile 


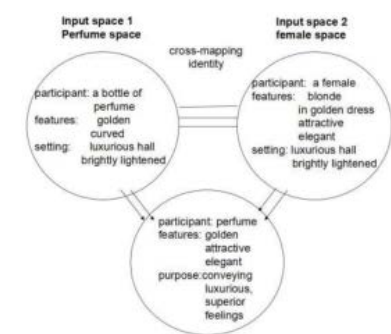

Figure 3 Conceptual integration of print perfume advertisements with vivid simile

Advertisements with mixed metaphor. Mixed metaphor refers to that target domain and source field integrate in a plane into an article, which is as shown in figure 4. In the advertisement, the target domain perfume and the source field flower are integrated into a bunch of flowers and its linguistic expression is "perfume is flower". Similarly, if we use conceptual metaphor and characters to express mixed metaphor, it cannot only fails to illustrate the inherent similarities, construction and new features generated in the process of deconstructing metaphor of perfume and flower but also fails to reveal differences of various visual metaphors. By use of the conceptual integration theory, the projection relation of the two input spaces is the relation of identity and causality. The abstract structure "flowers attracts hummers" in the input space of flowers and the features of flowers like "natural and harmonious" will be projected in the blend space. As an actor, the input space of perfume will be projected in the blend pace to form a new emergent structure) to express that perfume can attract others and enhance charm and also transfer the concepts of harmony, nature and attraction, which is as shown in figure 5.

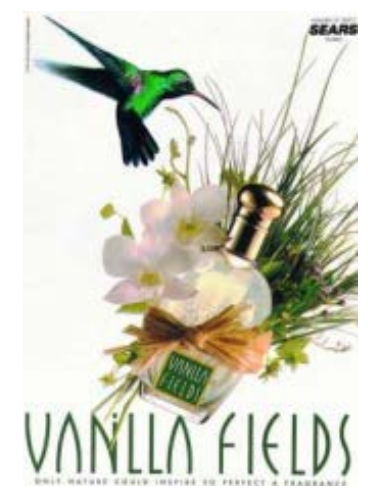

Figure 4 Advertisement with vivid metaphor

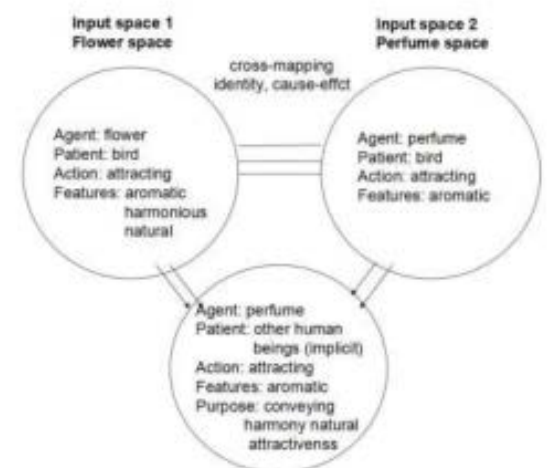

Figure 5 Conceptual integration of print perfume advertisements with mixed metaphor 1

As it is shown in figure 6, in the advertisement, the target domain perfume and the source field sakura are both reflected in the advertisement. However, images of the perfume and the sakura are integrated into a whole image through the visual effect of seeing sakura through the transparent bottle. In the mixed metaphor, the projection relation is very complicated, including identity, the whole-part relation and the causal relation. Identity refers to that perfume can maintain the transient beauty of sakura. In the process of generating the emergent structure, the input space 1 provides conceptual 
abstract structures and the input space 2 will provide elements and contents of the blend space, thus forming the psychological space of "perfume makes beauty eternal", which is as shown in figure 7.

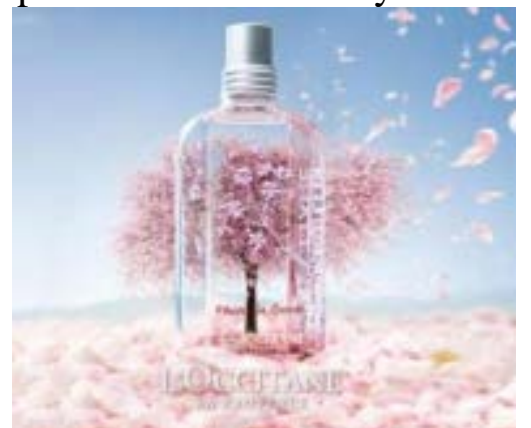

Figure 6 Advertisement with mixed metaphor

Figure 7 Conceptual integration 2 of print perfume advertisement with mixed metaphor

Advertisement with contextual metaphor. Print advertisements with contextual metaphor just represent target domain and source field can be deduced according to the background of the advertisement, which is as shown in figure 8. In the advertisement, only the target domain, perfume, and some water waves are represented, thus we can deduce the source field is ocean, lake or river constituted by water. Since the water waves are blue, the source field is most likely the ocean. Through identity projection, the input space 1 provides abstract structure to blend space and the input space 2 provides conceptual elements. After compression, there will be specific features within the emergent structure- to transfer concepts of nature, maturity and containment. The features come from integration of the two input spaces "for man" and the characters of ocean, deep and wide. The conceptual integration network is multiple dimensional since contextual metaphor involves the deducing process and the conceptual integration between water and ocean. And the other perfume advertisement also uses contextual metaphors, which is as shown in figure 10.

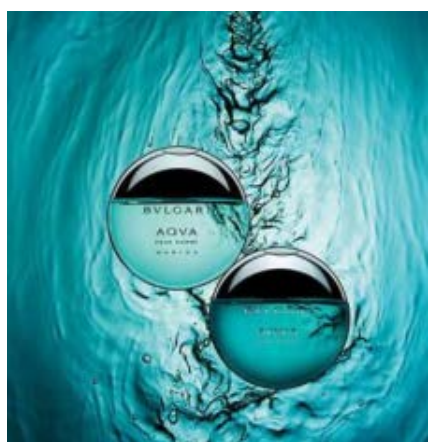

Figure 8 Advertisement with contextual metaphor 


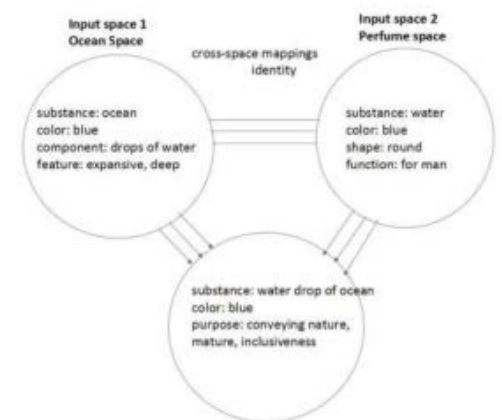

Figure 9 Analysis on conceptual integration of contextual metaphor 1

In the figure, there is only the target domain - perfume. However, the source field is not butterflies in the figure but flowers which attract them. Therefore, through deduction, we can know that the source field is flowers. And according to figure 11, we can know that the two input spaces have similar abstract structures, and the blend space also has the structure, inherent similarities of two input spaces and specific features of the input space 1, thus forming a new emergent structure. The contextual metaphor "transfer the product features of natural and attractive by using perfume attracting butterflies" also involves a multi-dimensional integration since the psychological space "butterflies are attracted by flowers" should be first constructed before the metaphor "perfume is flowers" is generated. Thus, the generation of contextual metaphor actually involves projection of several psychological spaces.

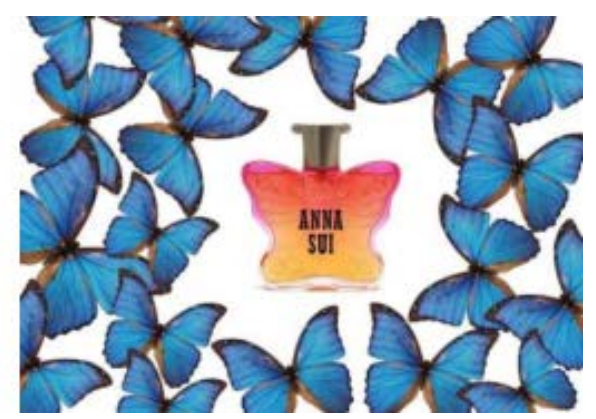

Figure 10 Advertisement with contextual metaphor

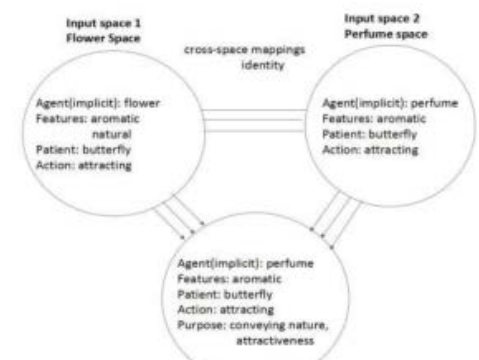

Figure 11 Analysis on conceptual integration of contextual metaphor 2

Advertisement with pure contextual metaphor. In the process of distinguishing visual metaphors, the writer discovered that metaphors adopted by some advertisements did not belong to the concentrated category proposed by Forceville. The metaphor is opposite to contextual metaphor, and the figure just represents source field without target domain, which is as shown in the figure. Therefore, the writer added a new metaphor category, which is called pure contextual metaphor, on the basis of the classification of Forceville. The conceptual integration of advertisements not only involves metaphor but also metonymy. As an ingredient of perfume, poppy replaces perfume to appear in the advertisement, which can be regarded as metonymy. And the two input spaces have inherent similarities - fragrant and curving. And the emergent structure of the blend space also has the specific features of the input space 1 - addictive and dangerous. The projection of the two input spaces reveals an asymmetry, which is as shown in figure 13. 


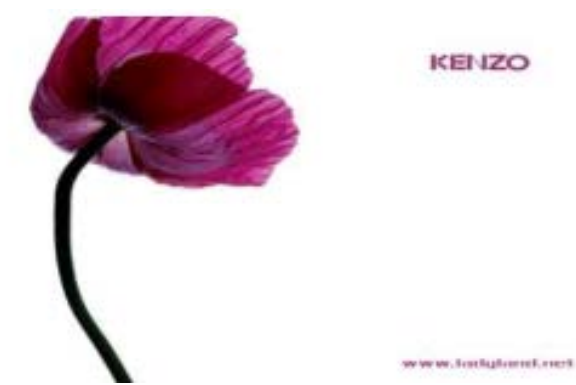

Figure 12 Advertisement with pure contextual metaphor

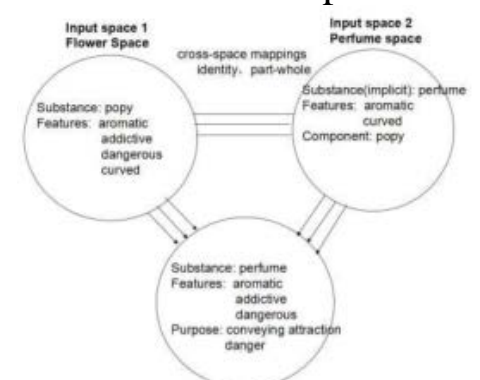

Figure 13 Analysis on conceptual integration of pure contextual metaphor

\section{Enlightenment of studies on visual metaphors}

Combining with the visual metaphor classification theory and the conceptual integration theory, the essay analyzed conceptual integration of various visual metaphor categories in print perfume advertisements and verified the superiority of the conceptual integration theory to explain metaphors. The conceptual integration theory cannot only explain the cognitive process of generation of metaphors but also detail mapping and projection relations between various psychological spaces and elements in the generating process of metaphors, thus it has a strong explanatory power. The conceptual integration network indicates that metaphor analysis of each advertisement involves projection of identity and inherent similarities of input spaces. The research discovered a new category of visual metaphor - pure contextual metaphor, which supplemented the visual metaphor theory and provide a reference to studies on visual metaphors in the future.

\section{References}

[1] Cui Shan, Qiu Zhijing. Application of conceptual grammatical metaphor in business English advertisements [J]. Journal of Mudanjiang Normal University (Philosophical Social Science Edition), 2014,05:103-104.

[2] Du Xiaozhuan. Study on metaphors in English advertisements [J]. Journal of Jilin Radio and Television University, 2013,07:70-71.

[3] Hu Weizhi. Study on metaphors in English advertisements from the perspective of the relevance theory [J]. Journal of Inner Mongolia Normal University (Education Science Edition), 2016,12:142-144.

[4] Zhao Na. Discussion on conceptual metaphors in English advertisements [J]. The Science Education Article Collects (first ten days), 2012,02:134+146.

[5] Wang Suping. Discussion on different functions of metaphors in English advertisements [J]. Journal of Pingxiang University, 2016,04:93-95. 